\title{
DOCUMENTACIÓN DE PROYECTOS EN LOS FABLABS: UN MODELO EXPLICATIVO
}

\author{
Project documentation in fablabs: \\ An explanatory model
}

\author{
Francisco-Javier Lena-Acebo y María-Elena García-Ruiz
}

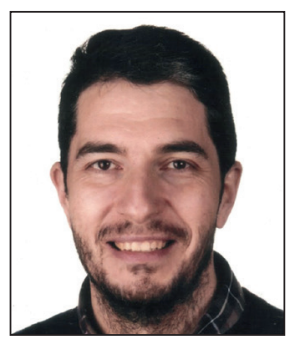

Francisco-Javier Lena-Acebo es profesor asociado LOU en el Departamento de Administración de Empresas de la Facultad de Económicas de la Universidad de Cantabria (UC). Doctor por la UC, licenciado en Ciencias Físicas (especialidad Electrónica) por la UC, Master Oficial en Empresa y Tecnología de la Información y Master E-business. Asimismo, es graduado en Psicología por la Universidad Isabel I (UI1) y Master Oficial en Prevención e Intervención Psicológica por la Universidad Internacional de Valencia (VIU). Miembro del grupo de investigación Atici, sus líneas de investigación incluyen los sistemas de información corporativos, la economía colaborativa, la innovación abierta, los nuevos modelos de negocio y la psicología del usuario.

https://orcid.org/0000-0002-7661-8398

lenafj@unican.es

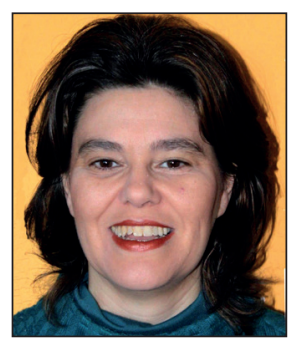

María-Elena García-Ruiz es profesora contratado-doctor en el Departamento de Administración de Empresas de la Facultad de Económicas de la Universidad de Cantabria (UC). Coordinadora del Master Oficial en Empresa y Tecnologías de la Información de la UC (MasterETI). Es miembro del grupo de investigación Atici de la UC y miembro fundador del Fablab Santander, así como directora del Master en Prototipado Rápido y Fabricación Digital (MasterFAB) impartido por la UC y el Fablab Santander. Entre sus líneas de investigación destacan las ecologías colaborativas, los nuevos modelos de negocio, la innovación abierta y la docencia basada en TIC.

https://orcid.org/0000-0002-8366-5678

elena.garcia@unican.es

Universidad de Cantabria Facultad de Ciencias Económicas Departamento de Administración de Empresas Avda. Los Castros s/n. 39005 Santander, España

\section{Resumen}

La actual expansión tecnológica ha permitido a una nueva generación de consumidores pasar de ser meros usuarios a artífices de sus propios bienes siguiendo la tendencia DIY (do it yourself). Los laboratorios de fabricación (fablab) potencian dicha tendencia y la complementan con la posibilidad de colaborar con otros usuarios (DIWO, do it with others), permitiendo, así mismo, replicar cualquier proyecto en otro fablab, ya que todos comparten dos elementos: maquinaria y aplicaciones open-source libres. Gracias a sus capacidades en la difusión del conocimiento a través de las redes, estos espacios amplían los límites de dicha colaboración a escala planetaria. La difusión de sus proyectos -que se muestra como uno de los pilares de este tipo de ecologías- requiere de una adecuada documentación de los mismos. En este artículo se identifican los elementos que influyen en el proceso de difusión realizada en los fablabs para obtener, finalmente, un modelo explicativo sobre la documentación de proyectos.

\section{Palabras clave}

Fablabs; Laboratorios de fabricación; Fabricación digital; Documentación; Proyectos; Entornos colaborativos; Código abierto; Open source; Innovación abierta.

\begin{abstract}
Current technological expansion has allowed a new generation of consumers to move from being mere users to architects of their own goods following the "do it yourself" (DIY) trend. Fabrication laboratories (fablabs) enhance this trend and complement it with the possibility of collaborating with other users (DIWO), allowing to replicate any project in another fablab, since they all share two elements: machines and free/open-source tools. Thanks to their capacities in the dissemination of knowledge through networks, these spaces expand the limits of such collaboration on a planetary scale. The diffusion of their projects - which is shown as one of the pillars of this ecology- requires an adequate documentation. This article identifies the elements that influence the diffusion process carried out in fablabs to obtain, finally, an explanatory model about project documentation.
\end{abstract}




\section{Keywords}

Fablabs; Fabrication laboratories; Digital fabrication; Documentation; Projects; Collaborative environments; Open source; Open innovation.

Lena-Acebo, Francisco-Javier; García-Ruiz, María-Elena (2018). “Documentación de proyectos en los fablabs: un modelo explicativo". El profesional de la información, v. 27, n. 4, pp. 830-839.

https://doi.org/10.3145/epi.2018.jul.12

\section{Introducción}

El actual cambio cultural en el desarrollo creativo, en el aprendizaje e incluso en la generación de prototipos como germen de productos tiene como referentes la cultura abierta, la colaboración social y el desarrollo de ecologías colaborativas en las que el aprendizaje se realiza mediante la transmisión de información entre colegas y la documentación abierta (Tabarés-Gutiérrez, 2016). En este contexto un grupo importante de espacios son los fablabs, en los que la corriente clásica de aprendizaje colaborativo se transforma desde el DIY (do it yourself) al DIWO (do it with others), convirtiéndose en elementos de innovación abierta que se incluyen en el proceso productivo industrial (Ruberto, 2015; Lô, 2017).

\section{Los fablabs transforman a sus usuarios de meros espectadores en protagonistas de la nueva revolución industrial}

El término fablab define una serie de espacios interconectados a través de una red internacional de colaboración, dotados de tecnología para la fabricación digital (Mikhak et al., 2002; Betts, 2010; Troxler; Schweikert, 2010; Troxler; Wolf, 2010), en los que se promueven actividades colaborativas, presentes habitualmente en ámbitos educativos, en contextos arquitectónicos e incluso en contextos médicos entre otros (Mikhak et al., 2002; Määttä; Troxler, 2011; Posch; Fitzpatrick, 2012; Mostert-Van-Der-Sar et al., 2013, Stephenson; Dow, 2014), mostrando su presencia también en las bibliotecas (Comalat-Navarra, 2015; De-Boer, 2015).

Se considera que la historia de los fablabs comienza de la mano de Neil Gershenfeld en el despliegue de la asignatura "How to make (almost) anything" en 2002 (Betts, 2010; Willemaerts et al., 2011; Gershenfeld, 2012; Posch; Fitzpatrick, 2012; Kohtala; Bosqué, 2013; George-Williams, 2015; Hielscher; Smith; Fressoli, 2015; Dreessen; Schepers; Leen, 2016). Esta asignatura desarrollada en el CBA (Center for Bits and Atoms) dependiente del MIT (Massachusetts Institute of Technology) y financiada por la NSF (National Science Foundation) (Tiala, 2011; Stephenson; Dow, 2014), necesitaba de un laboratorio en el que se pudieran generar y ensamblar materiales a pequeña escala (Cavalcanti, 2013; Walter-Herrmann; Büching, 2013; Capdevila, 2015; Hielscher; Smith; Fressoli, 2015). Este laboratorio aspiraba a transformarse en la punta de lanza de una revolución en el seno de la fabricación, liderando la transformación por medio de herramientas especializadas en la fabricación digital (Herrera-Polo; Juárez, 2012) que incluían desde impresoras 3D, cortadoras láser y control numérico para permitir la producción a pequeña escala (Tiala, 2011; Capdevila, 2015; Stephenson; Dow, 2014) aprovechando el funcionamiento de la web 2.0 como proceso democratizador del uso de la tecnología -en este caso, la tecnología de producción digital- por parte de los usuarios. Estos se convertían en prosumidores (Kotler, 1986; Ritzer; Dean; Jurgenson, 2012; Walter-Herrmann; Büching, 2013; Voigt; Montero; Menichinelli, 2016), pasando de meros espectadores a protagonistas (Gershenfeld, 2005; Kohtala; Bosqué, 2013).

A este primer fablab en el MIT le siguieron otros en Boston (2001, en el South End Technology Center de Tent City), en Costa Rica (2003, en el Instituto de Tecnología de Costa Rica), en India (2003, en la Science School Vugyan Ashram) y Ghana (2003, en el Takoradi Technical Institute), siendo el primero de Europa el Fablab Norway, en Lyngen, Noruega (Troxler; Wolf, 2010; Troxler, 2014; Hielscher; Smith; Fressoli, 2015), hasta alcanzar la cifra de 1.200 laboratorios en noviembre de 2017, de los cuales 47 se encuentran en territorio español.

Una de las ventajas de los fablabs es la capacidad que brindan a individuos, emprendedores y compañías, para transformar ideas de forma rápida en objetos físicos o prototipos de diseño mejorando el proceso de creación y desarrollo de elementos (Willemaerts et al., 2011; Büching; Walter-Herrmann; Schelhowe, 2012; Paio et al., 2012; Bosqué, 2013; Morel; Le-Roux, 2016).

La Fab Foundation (organización dependiente del programa Fablab del CBA del MIT encargada del apoyo a la expansión de los fablabs a nivel global) establece en su web cuatro criterios a partir de los cuales un laboratorio de fabricación digital puede considerarse verdaderamente un fablab y puede hacer uso del logo fablab en sus actividades:

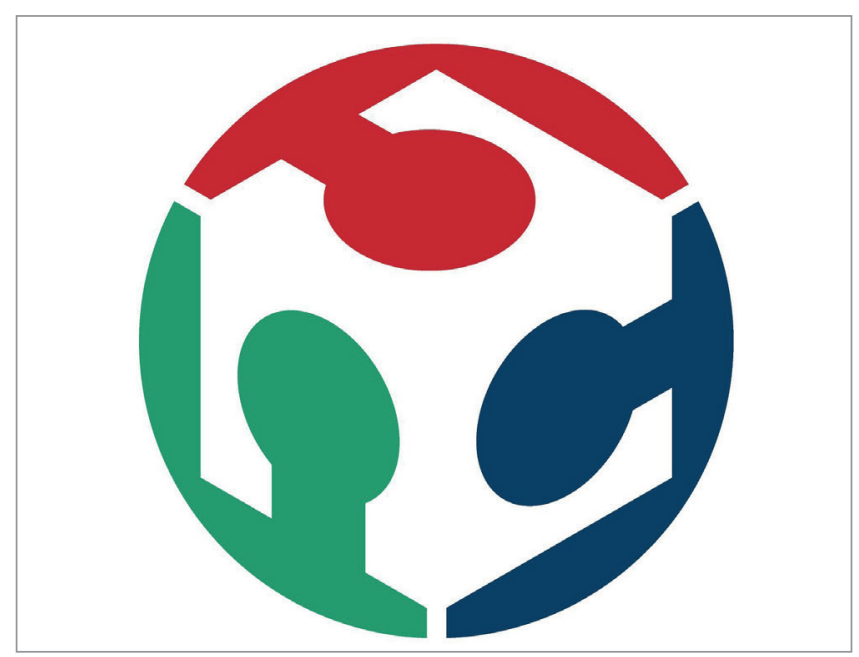

Logotipo fablab 
- Acceso público: se basan en un acceso democrático a las herramientas por lo que deben mostrarse abiertos al público de forma gratuita o con un sistema de pago por servicio al menos una vez al mes si es necesario (Kohtala; Bosqué, 2013).

- Adscripción a la Fab Charter (tabla 1), normas (Walter-Herrmann; Büching, 2013) que regulan todas las actividades a desarrollar, creadas en 2006 y modificadas en 2012, y que deben ser expuestas y suscritas por todo fablab (DeBoer, 2015; Kohtala, 2013; Hielscher; Smith; Fressoli, 2015).

- Disponibilidad de un conjunto de maquinaria común, enumerada en un listado disponible en la propia Fab Foundation, que permita la realización de los mismos proyectos en cualquiera de los fablabs existentes, de forma que a través de la distribución de conocimiento y la colaboración entre ellos, cualquier diseño realizado en un fablab, convenientemente documentado, pueda realizarse de forma satisfactoria en cualquier otro del mundo (Hielscher; Smith; Fressoli, 2015).

- Participación en la Fablab Network, la comunidad global de fablabs en la que se establece el intercambio de conocimiento entre ellos a través de videoconferencias, reuniones (eventos Fab), creación de workshops de forma conjunta o participación en el programa Fab Academy (Hielscher; Smith; Fressoli, 2015).

Los fablabs son, por tanto, piezas de una red global que conecta a usuarios interesados en la fabricación digital. Participan en el intercambio abierto de información de sus proyectos y diseños para convertir la red en una estructura sólida a través de la colaboración global, permitiendo el intercambio de información y fomentando el crecimiento económico (Diez, 2012). Todo ello dota a los procesos de documentación de los proyectos de una relevancia trascendental a nivel teórico.
No obstante, a pesar de ser una de las características principales y definitorias de los fablabs y estar recogida en su Fab Charter (tabla 1), la documentación de los proyectos es uno de sus principales problemas. No es la prioridad principal de sus usuarios, para quienes el proceso de documentación es una tarea poco divertida, consume demasiado tiempo y es difícil, a pesar de reconocerla como imprescindible para la generación de un conocimiento global (Wolf et al., 2013; García-Sáez, 2016). Éstos tienen como motivación principal para la participación en los fablabs la construcción y la experimentación, y no la documentación, que en algunos casos requiere de unas habilidades particulares que reconocen no disponer (Hielscher; Smith; Fressoli, 2015).

\section{El número de fablabs ha crecido vertigi- nosamente, de los tres laboratorios ini- ciales en 2003 a los 1.200 existentes en noviembre de 2017}

En contra de lo que pudiera pensarse, no existen estudios empíricos suficientes sobre la visión y las dificultades que los procesos de documentación de proyectos representan para los fablabs (Wolf et al., 2013), motivación principal para la realización del presente estudio.

\section{Metodología y desarrollo de la investigación.}

La rápida expansión de los fablabs posiblemente explique en parte la escasa bibliografía científica (Savastano et al., 2017) y la falta de modelos teóricos contrastados sobre los que basar investigaciones rigurosas. Estas dos carencias nos llevan a abordar nuestro trabajo a través de una doble metodología cualitativa y cuantitativa siguiendo un diseño de investigación adaptado al modelo exploratorio secuencial mixto (Creswell; Clark, 2007; Creswell, 2014).

Tabla 1. Fab Charter. Elaborado a partir de

http://www.fabfoundation.org/fab-labs/the-fab-charter

\footnotetext{
¿Qué es un fablab?

Los fablabs constituyen una red global de laboratorios locales que promueven la invención permitiendo el acceso a herramientas para la fabricación digital.

¿Que hay en un fablab?

Comparten un repertorio en evolución de capacidades básicas para hacer (casi) cualquier cosa para ser compartidas por proyectos y personas.

¿Qué proporciona la red de fablabs?

Asistencia operativa, educativa, técnica, financiera y logística más allá de lo que está al alcance de un único fablab.

¿Quién puede usar un fablab?

Están disponibles como recurso comunitario, ofreciendo acceso abierto a los individuos y acceso programado a los diferentes programas.

¿Cuáles son tus responsabilidades?

Seguridad: no dañar personas o máquinas.

Operaciones: ayudar con la limpieza, mantenimiento y mejora del lab.

Conocimiento: contribuir a la documentación y la instrucción.

¿A quién pertenecen los inventos realizados en un fablab?

Los diseños y los procesos desarrollados en los fablabs pueden ser protegidos y vendidos de la forma que el inventor elija, pero deberán estar disponibles para los usuarios para ser usados y aprender de ellos.

¿Cómo pueden usar el fablab los negocios?

Las actividades comerciales pueden prototiparse e incubarse en el fablab, pero no deberán entrar en conflicto con otros usuarios. Deberán desarrollarse más allá del fablab en lugar de dentro del fablab y se espera que beneficien a los inventores, los laboratorios y las redes de fablabs que contribuyeron a su éxito.

Fab Charter, Gershenfeld (2012), Fab Foundation
} 


\subsection{Fase cualitativa}

La fase inicial, cualitativa, incluyó la revisión y análisis de la bibliografía pertinente, la realización y posterior análisis de la información generada en grupos de discusión que contaron con la asistencia de expertos en fabricación digital, innovación abierta y miembros y gestores (fab managers) de fablabs, y la realización de 23 entrevistas semiestructuradas a managers, técnicos, responsables y directores de laboratorios de fabricación digital de diferentes puntos de España. El análisis cualitativo de la información generada en los anteriores procesos permitió la elaboración de hipótesis sobre los factores intervinientes en la documentación pública de proyectos en los fablabs. Además se empleó esta fase inicial para la construcción de un cuestionario, validado a través de un Delphi de varias etapas, que permitió la obtención de la información necesaria para la segunda fase.

\subsection{Fase cuantitativa}

La verificación de las hipótesis planteadas y la elaboración del modelo predictivo incluía la necesaria obtención de información de corte cuantitativo para lo que se empleó el cuestionario Fablab global survey, traducido a tres idiomas (español, inglés y francés), en el que se incluían 40 cuestiones agrupadas en tres bloques:

- descripción del fablab participante;

- descripción del modelo de negocio;

- procesos de documentación e innovación.

\section{A pesar de ser una de las características de los fablabs, la documentación de los proyectos no parece ser una tarea prio- ritaria}

Su distribución se realizó a través del correo electrónico dirigido a responsables y directores de fablabs en dos oleadas, solicitando de nuevo la colaboración a los laboratorios que no habían contestado transcurrido un mes desde la finalización de la primera. El resultado final contó con la participación de 124 laboratorios de fabricación digital (de los 445 listados en enero de 2016, año de aplicación), lo que supone una tasa de respuesta absoluta del $24,03 \%$, aunque debido a la rápida expansión de los fablabs y el tamaño de la muestra, nos obliga a tomar con cautela los resultados de este análisis.

\section{Resultados}

Debido a las características de este trabajo, los resultados incluyen por una parte la elaboración de las hipótesis de trabajo procedentes del estudio cualitativo y su validación a través del análisis cuantitativo, y por otra, el desarrollo y validación de un modelo predictivo sobre la documentación pública de proyectos.

\subsection{Evaluación de las hipótesis iniciales}

El análisis de la extensa documentación generada en los grupos de discusión, la revisión bibliográfica y las entrevistas en profundidad permitió la generación de diversas hipótesis sobre la documentación pública de proyectos en los fablabs, que se muestran en la tabla 2. 
Tabla 2. Hipótesis sobre la documentación pública de proyectos en los fablabs.

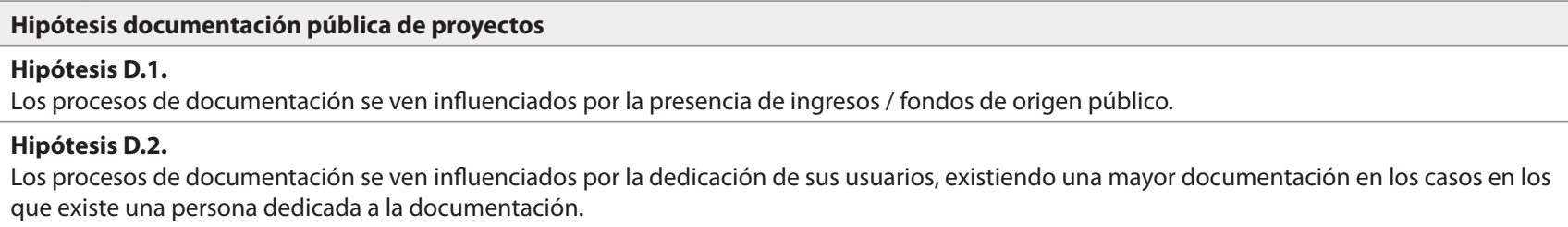
que existe una persona dedicada a la documentación.

H.D.2.1. Los procesos de documentación son mayores cuando el responsable de la documentación es el fab manager.

H.D.2.2. Los procesos de documentación son mayores cuando el responsable de la documentación es el líder o responsable del proyecto.

H.D.2.3. Los procesos de documentación son menores cuando no está definido un responsable de la documentación del proyecto.

Hipótesis D.3.

Los procesos de documentación se ven influenciados por las creencias acerca de la importancia relativa de la documentación.

\section{Hipótesis D.4.}

La participación en diferentes tipos de proyectos condiciona la documentación de los mismos:

H.D.4.1. El desarrollo de proyectos significativos en un fablab propicia la documentación.

H.D.4.2. La participación en proyectos conjuntos con otros laboratorios de la red fablab propicia la documentación de proyectos.

La evaluación de dichas hipótesis se realizó mediante la utilización de tablas de contingencia ${ }^{1}$ a partir de los datos generados en el cuestionario Fablab global survey, empleando como variable dependiente la existencia o no de una documentación superior al $50 \%$ de los proyectos realizados y como variables independientes las expresadas en la tabla 3.

"Pese a ser un fenómeno en auge, la importancia de los fablabs aún no ha tenido la repercusión necesaria en la bibliografía científica debido a su novedad

Los resultados (tabla 3) muestran cómo la existencia de un alto índice de proyectos documentados (un porcentaje superior al $50 \%$ ) se ve favorecida por la alta presencia de fondos públicos en la financiación de fablabs, así como el efecto positivo de la existencia de un responsable específico en la documentación asociado a la figura específica del fab manager, no siendo un factor relevante la responsabilidad del responsable o líder del propio proyecto en su documen- tación. También se comprueba el efecto negativo de la no existencia de un responsable de documentación definido. Por otro lado se evidencia el efecto positivo de la existencia de altas creencias sobre la importancia del proceso de documentación y la participación en proyectos significativos llevados a cabo por el propio fablab, descartando el efecto positivo que implica la participación en proyectos conjuntos con otros laboratorios de la red internacional de fablabs.

\subsection{Modelo explicativo}

Tras verificar las hipótesis, se planteó el modelo explicativo empleando una regresión logística binaria ${ }^{2}$ en la que una vez descartados los efectos de multi-colinealidad entre variables (cuyo significado se indica también en la tabla 3 ), se incluyen inicialmente las variables que mostraron correlación en el análisis anterior. Este modelo permite predecir la existencia o no de alta documentación de proyectos (superior al $50 \%$ de los proyectos realizados).

La bondad del modelo resultante se evaluó mediante el estadístico $\chi^{2}$ de Hosmer-Lemeshow, comprobando también la clasificación correcta del 92,3\% de los casos negativos y el $66,7 \%$ de los casos positivos, para lograr una capacidad

Tabla 3. Resultado de la evaluación de las hipótesis mediante tablas de contingencia

\begin{tabular}{|c|c|c|c|c|c|c|}
\hline \multicolumn{7}{|c|}{ Evaluación de las hipótesis mediante tablas de contingencia: resultados } \\
\hline \multicolumn{7}{|c|}{ Variable dependiente: Existencia de más del $50 \%$ de proyectos documentados } \\
\hline Hipótesis & Variable independiente & Variable & $\begin{array}{c}\text { Pearson } \\
\chi^{2}\end{array}$ & $\mathbf{P}$ & Phi & Evaluación \\
\hline H.D.1. & Alta presencia de fondos procedentes de fuentes públicas & Revpublbi & 4,755 & $0,029 *$ & 0,237 & Aceptada \\
\hline H.D.2.1. & Responsable de documentación: fab manager & Dorespfm & 6,215 & $0,013^{*}$ & 0,270 & Aceptada \\
\hline H.D.2.2. & Responsable de documentación: líder o responsable del proyecto & Doresplp & 0,071 & 0,790 & - & Rechazada \\
\hline H.D.2.3. & Responsable de documentación: No responsable & Doresnd & 3,902 & $0,048^{*}$ & $-0,214$ & Aceptada \\
\hline H.D.3. & Altas creencias sobre la importancia de la documentación & Agadvdoc & 12,795 & $0,000^{*}$ & 0,388 & Aceptada \\
\hline H.D.4.1. & Participación en proyectos significativos en el laboratorio & Proyfb & 8,707 & 0,003 & 0,320 & Aceptada \\
\hline H.D.4.2. & Participación en proyectos conjuntos con otros Lab de la red & Proyrd & 2,158 & 0,142 & - & Rechazada \\
\hline
\end{tabular}


Tabla 4. Estimación del modelo de regresión logística binaria para la variable Docuporbin

\begin{tabular}{|c|c|c|c|c|c|c|c|c|}
\hline \multicolumn{9}{|c|}{ Modelo de regresión logística binaria para la variable Docuporbin } \\
\hline & \multirow{2}{*}{ B } & \multirow{2}{*}{ E.T. } & \multirow{2}{*}{ Wald } & \multirow{2}{*}{ Gl } & \multirow{2}{*}{ Sig. } & \multirow{2}{*}{$\operatorname{Exp}(B)$} & \multicolumn{2}{|c|}{ I.C. $95 \%$ para Exp (B) } \\
\hline & & & & & & & Inferior & Superior \\
\hline Agadvdoc & 3,216 & 0,841 & 14,614 & 1 & 0,000 & 24,917 & 4,792 & 129,563 \\
\hline Proyfb & 2,605 & 0,736 & 12,523 & 1 & 0,000 & 13,530 & 3,197 & 57,258 \\
\hline Dorespfm & 1,450 & 0,675 & 4,621 & 1 & 0,032 & 4,265 & 1,137 & 16,005 \\
\hline Doresnd & $-1,667$ & 0,800 & 4,347 & 1 & 0,037 & 0,189 & 0,390 & 0,905 \\
\hline Constante & $-4,814$ & 1,125 & 18,328 & 1 & 0,000 & 0,008 & & \\
\hline \multicolumn{3}{|c|}{$\chi_{\text {Hosmer-Lemeshow }}^{2}=10,502(P=0,162)$} & \multicolumn{3}{|c|}{$\begin{array}{l}\text { Prueba Omnibus: } \chi^{2}=41,050(P=0.000) \\
-2 \text { Log Verosimilitud }=72,502\end{array}$} & & \multicolumn{2}{|c|}{$\begin{array}{l}\mathrm{R}_{\text {Coxy Snell }}^{2}=0,383 \\
\mathrm{R}_{\text {Nagelkerke }}^{2}=0,520\end{array}$} \\
\hline
\end{tabular}

de predicción total del $82,4 \%$. Así mismo se garantizó la significatividad positiva global del modelo a través de la prueba Omnibus $\chi^{2}=41,050(P=0,000)$. El modelo se mostró capaz de explicar el $52 \%$ de la varianza total.

$$
P(y=1)=\frac{1}{1+e^{[4,814-3,216 A G A D V D O C-2,605 P R O Y F B-1,450 D O R E S P F M+1,667 D O R E S N D]}}
$$

Ecuación 1. Función logística asociada al modelo explicativo

Atendiendo a los resultados del modelo, podemos afirmar que los fablabs que presenten una cultura con creencias favorables a la documentación presentarán una probabilidad de documentación 24,9 veces superior frente a los que no presenten esa marcada cultura. Análogamente, los fablabs que realicen proyectos significativos presentarán una probabilidad de documentación 13,53 veces superior a la probabilidad de no documentación. Además los que tengan como responsable de documentación dedicado al fab manager presentarán una probabilidad 4,26 superior a la probabilidad de no documentación frente a aquellos que no cuenten con dicho miembro dedicado. En el caso de la no existencia de una persona específica dedicada a la documentación, su relación es inversa y nos indica que la probabilidad de existencia de alta documentación por parte de un fablab en el que existe una persona específica destinada es 5,29 veces superior que en el caso en el que no exista una persona dedicada específicamente.

La probabilidad de documentación estará por tanto condicionada por los valores que individualmente adopten las variables independientes incluidas en el modelo, de forma que las probabilidades de que se muestre un alto porcentaje en la documentación de proyectos es inicialmente baja cuando:

- las creencias sobre la relevancia de la misma son bajas;

- no se desarrollan proyectos significativos;
- el fab manager no está encargado de la documentación de los proyectos;

- no hay una persona específicamente dedicada a la documentación ( $P=0,15 \%)$.

La probabilidad de que exista un elevado número de proyectos documentados es elevada cuando:

- existe una cultura sobre la relevancia de la documentación;

- se desarrollan proyectos significativos en el fablab;

- existe una persona dedicada a este aspecto $(P=73,21 \%)$, aumentando la probabilidad en el caso de que el fab manager se encargue de la documentación de los proyectos ( $P=92,11 \%)$.

Para evaluar la capacidad predictiva del modelo, se aplicó al caso particular de los laboratorios de fabricación digital españoles, alcanzando un elevado porcentaje de predicción $(94,1 \%)$, en consonancia con los resultados obtenidos en su elaboración.

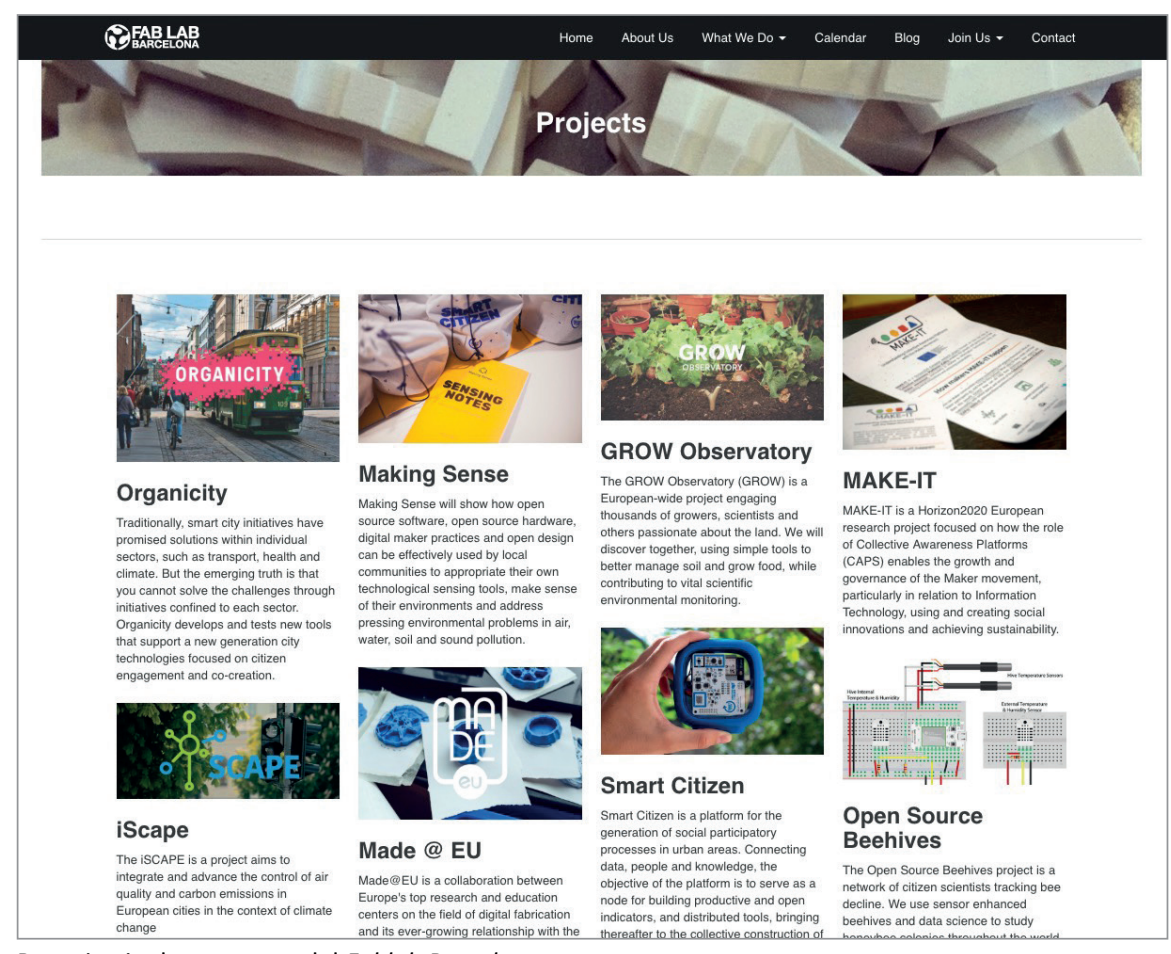

Repositorio de proyectos del Fablab Barcelona https://fablabbcn.org/projects.html 
Tabla 5. Evaluación del modelo propuesto para los fablabs españoles ordenados alfabéticamente

\begin{tabular}{|c|c|c|c|c|c|c|c|}
\hline \multicolumn{8}{|c|}{ Modelo de regresión logística para la variable Docuporbin } \\
\hline & \multicolumn{4}{|c|}{ Variables independientes } & \multicolumn{2}{|c|}{ Modelo } & \multirow{2}{*}{$\begin{array}{c}\text { Variable } \\
\text { Docuporbin }\end{array}$} \\
\hline & Agadvdoc & Proyfb & Dorespfm & Doresnd & Pred. & Calc. & \\
\hline Deusto Fablab & sí & no & no & sí & 0,04 & no & no \\
\hline Fablab Agrotech & sí & no & no & no & 0,17 & no & no \\
\hline Fablab Alicante & sí & sí & sí & no & 0,92 & sí & sí \\
\hline Fablab Barcelona & sí & sí & no & no & 0,73 & sí & sí \\
\hline Fablab Garaje 2.0 & sí & no & no & no & 0,17 & no & no \\
\hline Fablab IED Madrid & sí & no & no & no & 0,17 & no & no \\
\hline Fablab León & sí & no & no & no & 0,17 & no & sí \\
\hline Fablab Madrid CEU & sí & sí & no & no & 0,73 & sí & sí \\
\hline Fablab Palma & sí & sí & sí & no & 0,92 & sí & sí \\
\hline Fablab Sant Cugat & no & sí & no & no & 0,10 & no & no \\
\hline Fablab Santander & no & sí & no & no & 0,10 & no & no \\
\hline Fablab Sevilla & sí & sí & sí & sí & 0,69 & sí & sí \\
\hline Fablab Terrassa & no & sí & sí & no & 0,32 & no & no \\
\hline MADE Makerspace & no & sí & no & sí & 0,02 & no & no \\
\hline MedialabPrado Fablab & sí & sí & no & sí & 0,34 & no & no \\
\hline Smart Open Lab & sí & sí & no & no & 0,73 & sí & sí \\
\hline Tinkerers Lab & no & sí & sí & no & 0,32 & no & no \\
\hline
\end{tabular}

\section{Conclusiones}

La transmisión de conocimiento a través de la documentación pública de los proyectos realizados, a pesar de tratarse de uno de los procesos clave y característicos del entorno fablab, no parece realizarse con la frecuencia apropiada. Las dificultades que los laboratorios encuentran para la documentación de sus proyectos identificadas en la incipiente bibliografía científica, el análisis de los elementos que propician estos procesos de documentación no parece haber gozado del protagonismo suficiente. En este trabajo, y a la vista de los resultados obtenidos se puede argumentar la existencia de condicionantes que modulan la probabilidad de una alta documentación (superior al $50 \%$ de los proyectos realizados) en el seno de los fablabs.

Dicha probabilidad disminuye cuando no existe un responsable asignado al proceso de documentación de dichos proyectos y aumenta cuando existe:

- elevada financiación de las actividades del fablab a través de fondos públicos;

- fuerte implicación del fab manager como persona responsable de la documentación de los proyectos realizados;

- elevado nivel de acuerdo con la creencia de la importancia relativa del proceso de documentación de un proyecto como elemento de divulgación y generación de conocimiento;

- fuerte implicación de los usuarios en la realización de proyectos considerados como significativos en el propio fablab.

Desde este trabajo se plantea un modelo explicativo sobre la probabilidad de presentar una alta documentación de los proyectos realizados que incluye los efectos combinados de varias de estas situaciones, en concreto:

- existencia de una fuerte cultura sobre la documentación, vinculada a altas creencias sobre su importancia relativa;

- realización de proyectos significativos o considerados relevantes por los usuarios del laboratorio de fabricación digital;

- implicación del fab manager como responsable de la documentación de los proyectos que se llevan a cabo en el laboratorio;

- existencia de una relajación en la responsabilidad de la documentación de los proyectos que se realizan en el fablab (circunstancia que actuaría disminuyendo las probabilidades de existencia de alta documentación).

Dicho modelo muestra una alta capacidad predictiva y una gran adecuación al caso español, como se ha comprobado al evaluarse el modelo sobre 17 laboratorios de fabricación digital en territorio español.

A pesar de la colaboración de los laboratorios participantes en esta investigación es importante destacar que una de las mayores dificultades en las investigaciones relativas al entorno fablab y el resto de ecologías maker es precisamente su rápida expansión. El número de laboratorios crece de forma acelerada, lo que hace que la información sobre los mismos quede rápidamente sobrepasada y sea necesario actualizarla. Además, en un ambiente rápidamente cambiante como el tecnológico, tanto las técnicas como los materiales se actualizan de forma inexorablemente rápida, alterando los modelos de negocio y las actividades básicas y fundamentales de los laboratorios. Tal es el caso que, desde la propia Fab Foundation y la red internacional de fablabs, se realizan grandes esfuerzos para adecuar y consensuar las 
herramientas de comunicación en las que se engloban los procesos de documentación. Así, una vez caracterizados estos procesos como se ha realizado en el presente trabajo, posteriores líneas de investigación incluyen la validación del modelo planteado en diferentes agrupaciones de laboratorios para evaluar su validez global y estimar las posibles discrepancias existentes. El análisis pormenorizado de estas discrepancias podría apuntar a la existencia de factores culturales o regionales no contemplados en esta investigación.

Finalizamos, por tanto, haciendo hincapié en la importancia de la documentación de los proyectos como característica básica de los fablabs frente a otras agrupaciones. Aunque en el entorno maker en el que se integran existe una clara predisposición al intercambio de información y al aprendizaje entre pares, los fablabs se precian de ser elementos que emplean su red internacional y el uso de un conjunto de máquinas estándar para replicar cualquier proyecto en cualquier lugar del mundo, extendiendo esta posibilidad y generando una especial necesidad de documentación en medios adecuados. Un proceso que, a pesar de las dificultades que requiere su realización, está generalmente bien valorado entre los usuarios, es apreciado por la comunidad y constituye el verdadero pilar del aprendizaje entre iguales y el punto de partida a una innovación abierta que incluya, en las ecologías generadas, los entornos empresariales e industriales.

\section{Notas}

1. Las tablas de contingencia son utilizadas como una técnica estadística que emplea la tabulación cruzada de datos de variables dependientes e independientes -admitiendo de diferentes tipos, incluso nominales o cualitativas-, permitiendo medir la asociación entre ellas y evaluar la significatividad de dicha dependencia. En el empleo de tablas de contingencia, el estadístico Phi permite evaluar el grado de asociación entre variables -especialmente aplicado para tablas de $2 \times 2-$, oscilando su valor entre 0 y 1 . La significatividad del estadístico se evalúa a través de la prueba Chi-Cuadrado de Pearson, que permitirá aceptar o rechazar la hipótesis de independencia (Hair et al., 1999).

2. La regresión logística binaria es una técnica estadística de análisis de datos empleada para relacionar una variable dependiente cualitativa con una o más variables que se consideran independientes permitiendo, incluso, contrastar hipótesis. En las regresiones logísticas binarias se emplea la razón de odds u odds ratio - $\operatorname{Exp(B)-que,~entre~otros~signi-~}$ ficados, también se emplea como medida de la magnitud de la asociación entre las variables dependiente e independiente siendo una relación positiva y directa cuando el valor es mayor que 1 y una relación negativa o inversa cuando su valor es inferior a 1. Una odds ratio igual a 1 indicaría la ausencia de relación entre las variables (Salas-Velasco, 1996; Hair et al., 1999).

\section{Referencias}

Betts, Brian (2010). "Bringing the factory home (personal fabrication technology)". Engineering \& technology, v. 5, n. 8, pp. 56-58.

https://doi.org/10.1049/et.2010.0813

Bosqué, Camile (2013). "Fablabs: La démocratie du design". Architecture d'aujourd hui, n. 398, pp. 70-77.

http://www.camillebosque.com/larchitecture-daujourdhui

Büching, Corinne; Walter-Herrmann, Julia; Schelhowe, Heidi (2012). "The C-model of interaction between humans and digital media in learning environments". En: $15^{\text {th }}$ Intl conf on interactive collaborative learning (ICL), pp. 1-7. https://doi.org/10.1109/ICL.2012.6402152

Capdevila, Ignasi (2015). "Coworking spaces and the localized dynamics of innovation in Barcelona". Journal of innovation management, v. 19, n. 3.

https://doi.org/10.2139/ssrn.2502813

Cavalcanti, Gui (2013). "Is it a hackerspace, makerspace, techShop, or fablab?". Make magazine, June $22^{\text {nd }}$.

https://makezine.com/2013/05/22/the-difference-betweenhackerspaces-makerspaces-techshops-and-fablabs 
Comalat-Navarra, Maite (2015). “Bibliotecas públicas e impresoras 3D: el debate está servido". El profesional de la información, v. 24, n. 2, pp. 185-192.

https://doi.org/10.3145/epi.2015.mar.12

Creswell, John W. (2014). Research design: Qualitative, quantitative, and mixed methods approaches. Thousand Oaks: Sage Publications. ISBN: 9781412965576

Creswell, John W.; Plano-Clark, Vicky (2007). Designing and conducting mixed-methods research. Thousand Oaks: Sage. ISBN: 9781412927919

De-Boer, Jeroen (2015). "The business case of FryskLab, Europe's first mobile library fablab". Library hi tech, v. 33, n. 4, pp. 505-518.

https://doi.org/10.1108/LHT-06-2015-0059

Diez, Tomás (2012). "Personal fabrication: Fablabs as platforms for citizen-based innovation, from microcontrollers to cities". Nexus network journal, v. 14, n. 3, pp. 457-468. https://doi.org/10.1007/s00004-012-0131-7

Dreessen, Katrien; Schepers, Selina; Leen, Danny (2016). "From hacking things to making things. Rethinking making by supportin non-expert users in a fablab". Interaction design and architecture(s) journal (IxD\&A), n. 30, pp 47-64. https://goo.gl/wgc6kB

García-Sáez, César (2016). (Casi) todo por hacer. Una mirada social y educativa sobre los fab labs y el movimiento maker. Fundación Orange.

https://goo.gl/SbJijc

George-Williams, Silvya-Caroline (2015). "If you build it will they come?: Building a fablab in the University of Texas @ Arlington Libraries and building faculty partnerships for its use". In: $122^{\text {nd }}$ ASEE Annual conference \& exposition, June 14-17, Seattle, WA.

https://www.asee.org/public/conferences/56/papers/11197/ download

Gershenfeld, Neil (2005). Fab: Personal fabrication, fab labs, and the factory in your computer. New York: Basic Books Inc. ISBN: 9780465027460

Gershenfeld, Neil (2012). "How to make almost anything: The digital fabrication revolution". Foreign affairs, v. 91, n. 6, pp. 43-57.

http://cba.mit.edu/docs/papers/12.09.FA.pdf

Hair, Joseph F.; Anderson, Rolph.; Tatham, Ronald L.; Black, William C. (1999). Análisis multivariante. Madrid: Prentice-Hall. ISBN: 9788483220351

https://wwwyyy.files.wordpress.com/2018/01/anc3a1lisismultivariante-de-joseph-f-hair-y-otros.pdf

Herrera-Polo, Pablo; Juárez, Benito (2012). "Perspectivas en los laboratorios de fabricación digital en Latinoamérica". En: Proceedings of the $16^{\text {th }}$ Iberoamerican congress of digital graphics graphics, pp. 285-289.

https://repositorioacademico.upc.edu.pe/handle/10757/604776

Hielscher, Sabine; Smith, Adrian; Fressoli, Mariano (2015). WP4 Case study report: FabLabs, report for the Transit FP7 project. UK: SPRU, University of Sussex Brighton. https://goo.gl/D3xkCF
Kohtala, Cindy (2013). "Shaping sustainability in fablab". En: Melkäs, Helinä; Buur, Jens (eds.). Proceedings of the Participatory innovation conf PIN-C 2013, LUT Scientific and Expertise Publications, Lappeenranta University of Technology. Lahti, Finland, pp. 287-290.

https://blogs.aalto.fi/makerculture/files/2013/05/pin-c2013-Kohtala_SustainabilityFabLabs.pdf

Kohtala, Cindy; Bosqué, Camile (2013). "The story of MIT-Fablab Norway: Community embedding of peer production". Journal of peer production, n. 5. https://goo.gl/EJd1hF

Kotler, Philip (1986). "The prosumer movement: A new challenge for marketers". Advances in consumer research, v. 13, n. 1, pp. 510-513.

http://acrwebsite.org/volumes/6542/volumes/v13/NA-13

Lô, Amadou (2017). “Un fablab d'entreprise pour favoriser l'ambidextrie des salariés. Étude de cas chez Renault". Revue française de gestion, v. 43, n. 264, pp 81-99. https://doi.org//10.3166/rfg.2017.00113

Määttä, Anu; Troxler, Peter (2011). "Developing open \& distributed tools for fablab project documentation". In: Procs of the $6^{\text {th }}$ Open knowledge conf.

http://ceur-ws.org/Vol-739/paper_21.pdf

Mikhak, Bakhtiar; Lyon, Christofer; Gorton, Tim; Gershenfeld, Neil; McEnnis, Caroline; Taylor, Jason (2002). "Fab lab: an alternate model of ICT for development". En: $2^{\text {nd }}$ Int conf on open collaborative design for sustainable innovation. Development by design.

https://goo.gl/DsrbnW

Morel, Laure; Le-Roux, Serge (2016). Fab labs. Innovative user. Hoboken, USA: John Wiley \& Sons - ISTE. ISBN: 9781 848218727

https://doi.org/10.1002/9781119318392

Mostert-Van-Der-Sar, Manon; Mulder, Ingrid; Remijn, Leo; Troxler, Peter (2013). "Fablabs in design education". In: Procs of the $15^{\text {th }}$ Intl conf on engineering and product design education: Design education - Growing our future, 5-6 September, Dublin Institute of Technology, Dublin, Ireland, pp. 629-634

https://www.designsociety.org/download-publication/34781/ fablabs_in_design_education

Paio, Alexandra; Eloy, Sara; Rato, Vasco; Resende, Ricardo; De-Oliveira, Maria-João (2012). "Prototyping vitruvius, new challenges: Digital education, research and practice". Nexus network journal, v. 14, n. 3, pp. 409-429.

https://doi.org/10.1007/s00004-012-0124-6

Posch, Irene; Fitzpatrick, Geraldine (2012). "First steps in the fablab". In: Procs of the $24^{\text {th }}$ Australian computer-human interaction conf - OzCHI'12. ACM Press, pp. 497-500. https://publik.tuwien.ac.at/files/PubDat_215671.pdf

Ritzer, George; Dean, Paul; Jurgenson, Nathan (2012). "The coming of age of the prosumer". American behavioral scientist, v. 56, n. 4, pp. 379-398.

https://doi.org/10.1177/0002764211429368

Ruberto, Francesco (2015). "Fablabs as new innovation infraestructure for the Italian industry". IOSR Journal of 
busines and management, v. 15, n. 3, pp. 80-87. http://iosrjournals.org/iosr-jbm/papers/Vol17-issue3/ Version-3/L017338087.pdf

Salas-Velasco, Manuel (1996). “La regresión logística. Una aplicación a la demanda de estudios universitarios”. Estadística española, v. 38, n. 141, pp. 193-217.

https://goo.gl/hk9xxj

Savastano, Marco; Bellini, Francesco; D’Ascenzo, Fabrizio; Scornavacca, Eusebio (2017). "Fablabs as platforms for digital fabrication services: A literature analysis". Lecture notes in business information processing, v. 279, pp. 24-37. https://doi.org/10.1007/978-3-319-56925-3_3

Stephenson, Makeda; Dow, Douglas (2014). "The community FabLab platform: Applications and implications in biomedical engineering". En: $36^{\text {th }}$ Annual intl conf of the IEEE Engineering in Medicine and Biology Society, pp. 1821-1825. https://doi.org/10.1109/EMBC.2014.6943963

Tabarés-Gutiérrez, Raúl (2016). "Approaching maker's phenomenon". Interaction design and architerure(s) journal - IX$D \& A$, n. 30, pp. 19-29.

https://www.researchgate.net/publication/312606673_ Approaching_makers_phenomenon

Tiala, Silvia (2011). "Fablabs: Re-envisioning innovation and 'entrepreneering'". ASQ Higher education brief, v. 4, n. 5. https://goo.gl/hHab2m

Troxler, Peter (2014). "Fab labs forked: A grassroots insurgency inside the next industrial revolution from a book programmes national". Journal of peer production, n. 5. https://goo.gl/8TaHbJ
Troxler, Peter; Schweikert, Simon (2010). "Developing a business model for concurrent enterprising at the fablab". En: Procs of the $16^{\text {th }}$ Intl conf on concurrent enterprising, pp. 14-21.

https://doi.org/10.1109/ICE.2010.7476996

Troxler, Peter; Wolf, Patricia (2010). "Bending the rules: The fablab innovation ecology". En: $11^{\text {th }}$ Intl CINet conf. Zurich, Switzerland, 5-7 September.

https://goo.gl/N6tTi3

Voigt, Christian; Montero, Calking-Suero; Menichinelli, Massimo (2016). "An empirically informed taxonomy for the

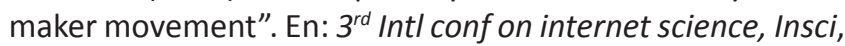
pp. 189-204.

https://goo.gl/Juw2dg

https://doi.org/10.1007/978-3-319-45982-0_17

Walter-Herrmann, Julia; Büching, Corinne (2013). Fablab: of machines, makers and inventors. Wetzlar: [Transcript] Cultural and Media Studies. ISBN: 9783873623826

Willemaerts, Christe; Dewulf, Wlim; Lambaerts, Marc; Boeykens, Stefan; Vanden, Vero; Van-Aken, Jeroen; Pelsmaekers, Koen; Voet, André; Lauwers, Bert (2011). "Introducing engineering students to manufacturing in a fabrication laboratory (fab lab)". In: Procs of the World engineering education flash week, pp. 72-79.

https://goo.gl/4TBbVw

Wolf, Patricia; Troxler, Peter; Kocher, Pierre-Yves; Harboe, Julie; Gaudenz, Urs (2013). "Sharing is sparing: Open knowledge sharing in fablabs". Journal of peer production, n. 5. https://goo.gl/PVUTG4

\section{5 ฐँचISIS IWETEL Foro para profesionales de https://www.rediris.es/list/info/iwetel.html bibliotecas y documentación}

Con unos 6.000 miembros, IweTel es la mayor lista de distribución en castellano para debatir y estar al día sobre temas de biblioteconomía y documentación.

Fue creada en 1993 por Tomàs Baiget, como complemento de Information World en Español (IWE), revista que en 1999 pasó a denominarse El profesional de la información (EPI).

Desde 1998 IweTel está alojada en el servicio de listas de RedIRIS, siendo posible consultar en sus archivos estos 18 años de la historia de la documentación en España:

https://listserv.rediris.es/cgi-bin/wa?A0=IWETEL

La lista cuenta con 4 moderadores que permanentemente filtran los mensajes para evitar spam, men-

sajes inapropiados, anuncios, mensajes repetidos, etc.:

David Gómez (Observatorio de la Infancia de Andalucía),

Isabel Olea (EPI, León),

Julio Alonso-Arévalo (Universidad de Salamanca),

Tomàs Baiget (EPI, Barcelona).

Puedes suscribirte a IweTel en:

https://listserv.rediris.es/cgi-bin/wa?SUBED1=IWETEL\&A=1 